\title{
Rehabilitation after coronary artery by-pass grafting and improved quality of life
}

\author{
N. A. Hoad, MA, MRCP and I. C. Crawford, CBE, FRCP \\ Queen Elizabeth Military Hospital, Woolwich, London SE18
}

\begin{abstract}
Long wating lists for coronary artery by-pass grafting often mean that patients have to endure a prolonged period of pre-operative disability, a known poor prognostic factor for their future quality of life and employment prospects. A survey of 60 patients who attended a post-operative rehabilitation course designed to restore their fitness and self-esteem, and hence their quality of life, has shown encouraging results. When questioned a mean 16 months later, 85 per cent are working and significant improvements have been seen in all four quality of life indicators assessed: job satisfaction, family life, social life and sex life. Significant improvements are also seen in their symptoms. Cardiac rehabilitation should be made more available following coronary artery by-pass grafting.
\end{abstract}

Keywords: Coronary artery by-pass grafting, cardiac rehabilitation, quality of life

\section{Introduction}

While coronary artery by-pass grafting (CABPG) effectively relieves angina and may prolong life in certain anatomical patterns of coronary artery disease, less is known about to what extent quality of life can be improved ${ }^{1}$. The use of return-to-work rates as an indicator has given variable results ${ }^{2}$. A recent study from Oxford has shown little change in a number of quality of life indices, despite good symptom control ${ }^{3}$. Neither is much known about the role of cardiac rehabilitation in improving the quality of life in such patients ${ }^{4}$.

The aim of this study was to determine whether participation in a well-established cardiac rehabilitation course can improve return-to-work rates and quality of life in patients who had recently undergone CABPG. The course at this hospital began in 1979 with the intention of restoring patients with ischaemic heart disease to such physical and psychological fitness as would enable them to return to full time employment. Over the years, an increasing proportion, now over one third, have undergone CABPG. Of them, over 80 per cent are civilian patients.

Address for correspondence: Major N. A. Hoad, Queen Elizabeth Military Hospital, Stadium Road, Woolwich, London SE18, UK

(C) 1990 Butterworth-Heinemann Ltd 0306-4179/90/020120-03

\section{Patients and methods}

Between September 1986 and May 1988, 79 patients who had recently undergone CABPG began a cardiac rehabilitation course at this hospital. They had been referred from a number of cardiac surgical units. Our only exclusion criteria were age over 65 and significant non-cardiovascular disability. Our preferred time for accepting them was eight to ten weeks post-operatively to allow for good sternal healing.

After initial history and examination, all underwent maximal exercise testing on a treadmill using the standard Bruce protocol. Those unable to complete three minutes for any reason were not permitted to continue, and neither were those who developed complex arrhythmias or chest pain accompanied by significant ST segment depression. The maximum heart rate achieved by each patient was used as a baseline for his training programme.

The course itself consisted of three five day residential blocks. During each block the patients exercised to an increasing proportion of their maximum heart rate, from 70 per cent in block 1 to 85 per cent in block 3 . This was achieved during short high-intesnsity sessions of aerobic exercise such as swimming, circuit training and jogging. Longer sessions of low intensity endurance exercise such as walking and cycling were also performed. The patients, up to 12 at any one time, were supervised throughout by a nurse and two physiotherapists, and medical assistance was always available.

The exercise training was combined with an educational and counselling programme which included group discussions with a physician, dietician, psychologist and physiotherapist. At the end of the course the patients were interviewed individually, ideally with their partner. They were encouraged to continue with a modified exercise programme and, with the life style changes recommended by us, to promote the secondary prevention of ischaemic heart disease. After one review here they returned to the care of their referring hospital.

All the above patients who were able to complete the course were sent a questionnaire designed to assess their symptoms and quality of life at three specific times: immediately before their operation, on arrival for rehabilitation, and sixteen months later. The symptoms chosen were angina, breathlessness, physical limitation and psychological limitation. The 
quality of life indicators were job satisfaction, family life, social life and sex life. Their employment status was also assessed, including that before the onset of their ischaemic heart disease.

The possible answers and the scoring system used were as follows. For symptoms: nil (0), mild (1), moderate (2), severe (3). For quality of life: excellent $(0)$, good (1), average (2), poor (3). The mean scores for each assessment were tested for significance by a two-tailed $t$ test.

\section{Results}

Of the original 79 patients, three (3.8 per cent) did not commence the training programme because of an abnormal exercise test. Of the remaining 76 , four $(5.3$ per cent) could not complete the course because of musculo-skeletal injuries, and one (1.3 per cent) because of ventricular arrhythmias. The study population therefore consisted of 71 men, mean age $48.4 \mathrm{yr}$ (SD 7.0), from whom 60 replies to the questionnaire were received, a response rate of 84.5 per cent. They had attended the course for a mean 16.3 (SD7.1) months previously.

\section{Employment}

Before symptoms began, 52 of the 56 patients ( 93 per cent) were working, two (3.6 per cent) were retired, and two (3.6 per cent) were unemployed. Preoperatively, 42 (75 per cent) were still at work, five (8.9 per cent) at a reduced level. On arrival here, 31 (55 per cent) were back at work, seven ( 23 per cent) at a reduced level, and 16 (29 per cent) were still on sick leave. Now, 47 of the 58 (81 per cent) are at work, five

Table 1. Symptom scores

\begin{tabular}{|c|c|c|c|}
\hline & Preoperation & $\begin{array}{l}\text { Prerehabili- } \\
\text { tation }\end{array}$ & $\begin{array}{c}16 \text { months } \\
\text { post-op. }\end{array}$ \\
\hline \multicolumn{4}{|l|}{ Angina } \\
\hline Mean score (SD) & $1.81(1.16)$ & $0.70(0.87)$ & $0.31(0.62)$ \\
\hline Change & - & 1.11 & 0.39 \\
\hline Significance & - & $* * *$ & $* *$ \\
\hline$n$ & 58 & 56 & 59 \\
\hline \multicolumn{4}{|l|}{ Breathlessness } \\
\hline Mean score (SD) & $1.55(1.20)$ & $0.61(0.77)$ & $0.41(0.59)$ \\
\hline Change & - & 0.94 & 0.20 \\
\hline Significance & - & $* * *$ & NS \\
\hline$n$ & 58 & 57 & 59 \\
\hline \multicolumn{4}{|l|}{ Physical limitation } \\
\hline Mean score (SD) & $1.98(0.96)$ & $1.18(0.82)$ & $0.45(0.68)$ \\
\hline Change & - & 0.80 & 0.73 \\
\hline Significance & - & $* * *$ & $* * *$ \\
\hline$n$ & 58 & 55 & 58 \\
\hline \multicolumn{4}{|c|}{ Psychological limitation } \\
\hline Mean score (SD) & $1.60(1.14)$ & $1.09(0.94)$ & $0.47(0.65)$ \\
\hline Change & - & 0.51 & 0.62 \\
\hline Significance & - & $* *$ & $* * *$ \\
\hline$n$ & 58 & 56 & 58 \\
\hline
\end{tabular}

Significance levels for tables 1 and 2:

* significant at $5 \%$ level

** significant at $1 \%$ level

*** significant at $0.1 \%$ level
(11 per cent) at a reduced level, five (8.6 per cent) are retired, and six (10 per cent) are unemployed. Overall, 84 per cent of those working before their symptoms began are now still working.

\section{Symptoms}

The results shown in Table 1 confirm that CABPG is effective in relieving angina and breathlessness. After rehabilitation however, greater further improvements are seen in the perceptions of their physical and psychological limitations than in their angina and breathlessness scores. This may indicate an increased confidence in their abilities.

\section{Quality of life}

The results shown in Table 2 indicate a significant improvement in only one of the four indices in the first eight to ten weeks after CABPG. At 16 months, all show a significant improvement over the prerehabilitation assessment.

\section{Discussion}

The definition of a successful outcome after CABPG has moved beyond prolongation of life and relief from angina and should now include quality of life improvements, of which only one, return to work rates, has been studied in detail ${ }^{2}$. Symptomatic relief and quality of life improvements are by no means synonymous ${ }^{3}$.

Return to work rates vary in other series ${ }^{2,5,6}$, but are still not as good as might be expected, given the excellent symptomatic relief gained by almost all the patients. Analysis of these results has suggested that the risk of later unemployment is strongly related to the length of time spent disabled and hence unable to

Table 2. Quality of life scores

\begin{tabular}{|c|c|c|c|}
\hline & Preoperation & $\begin{array}{c}\text { Prerehabili- } \\
\text { tation }\end{array}$ & $\begin{array}{l}16 \text { months } \\
\text { post-op. }\end{array}$ \\
\hline \multicolumn{4}{|l|}{ Job satisfaction } \\
\hline Mean score (SD) & $1.78(1.11)$ & $1.52(0.98)$ & $1.04(0.92$ \\
\hline Change & - & 0.26 & 0.48 \\
\hline Significance & - & NS & $* *$ \\
\hline$n$ & 54 & 44 & 55 \\
\hline \multicolumn{4}{|l|}{ Family life } \\
\hline Mean score (SD) & $1.07(0.83)$ & $0.91(0.67)$ & $0.62(0.62)$ \\
\hline Change & - & 0.16 & 0.29 \\
\hline Significance & - & NS & $*$ \\
\hline$n$ & 56 & 55 & 58 \\
\hline \multicolumn{4}{|l|}{ Social life } \\
\hline Mean score (SD) & $1.89(0.97)$ & $1.54(0.82)$ & $1.05(0.76)$ \\
\hline Change & - & 0.25 & 0.49 \\
\hline Significance & - & $*$ & $*$ \\
\hline$n$ & 56 & 54 & 58 \\
\hline \multicolumn{4}{|l|}{ Sex life } \\
\hline Mean score (SD) & $2.11(0.89)$ & $1.88(0.90)$ & $1.35(0.91)$ \\
\hline Change & - & 0.23 & 0.53 \\
\hline Significance & - & NS & $* *$ \\
\hline$n$ & 53 & 52 & 54 \\
\hline
\end{tabular}


work pre-operatively ${ }^{6}$. This may help to explain the poor results in the UK, where in many regions the waiting list for CABPG is extremely long.

Rehabilitation after CABPG, well established in America and some European countries ${ }^{4,7}$, has been received with mixed enthusiasm in the $\mathrm{UK}^{8,9}$. Perhaps this is because doubts persist about whether rehabilitation, aimed at restoring fitness and promoting secondary prevention of ischaemic heart disease by life style changes, can influence the long term prognosis ${ }^{10}$. What is known, and more importantly in this context, is that a significant improvement in fitness can be safely achieved ${ }^{11,12}$. This should help patients to recover their self-esteem, renounce the sick role more quickly and return to work ${ }^{13}$. This would be especially valuable in the United Kingdom, for the reasons described above.

We believe that the combination of an exercise programme with education about the secondary prevention of ischaemic heart disease, with many opportunities for discussing problems with both staff and fellow patients, should maximise the potential quality of life benefits that surgery can provide. Indeed, the Consensus Development Conference on CABPG stated that 'the full benefits of surgery require counselling and rehabilitation measures 1 , advice which is reiterated in a recent review of long term management after $\mathrm{CABPG}^{13}$.

Our results support this belief. We chose four simple and wide-ranging quality of life indicators, suitable for inclusion in a self-reported questionnaire, because we agree that the patients' own judgement of the efficacy of their treatment is important ${ }^{5}$. The results based on an 85 per cent response rate, which should be large enough to enable valid conclusions to be drawn, are encouraging with all four showing a significant improvement after rehabilitation. This improvement is sustained, at least in the medium term, with no difference being seen between the scores of those who attended six or 24 months previously. A similar pattern of sustained improvement was seen in the symptom scores.

Return to work rates compare well with a recent British series ${ }^{3}$, and also with a major American series $^{14}$. Overall, we believe that cardiac rehabilitation helps maximize the symptomatic and quality of life benefits of CABPG, and should be made more available, especially to show who have suffered a long period of pre-operative disability. A residential course is expensive, but Bethell ${ }^{15}$ has shown that it is feasible to set up and run an out-patient rehabilitation course safely and inexpensively. This is probably the answer for the future.

\section{References}

1 Consensus Development Conference: Coronary artery by-pass grafting $B M J$ 1984, 289, 1527-1529

2 Russell, R.O., Abi-Mansour, P. and Wenger, N.K. Return to work after coronary by-pass surgery and percutaneous transluminal angioplasty. Issues and potential solutions Cardiology 1986, 73, 306-322

3 Mayou, R. and Bryant, B. Quality of life after coronary artery surgery QJMed 1987, 63(239), 239-248

4 Froelicher, P., Jensen, D. and Sullivan, M. A randomized trial of the efficacy of exercise training after coronary artery by-pass surgery Arch Intern Med $1985,145,689-692$

5 Konttingen, M.P. Long-term efficacy of coronary artery by-pass surgery Scan I Thor Cardiovasc Surg 1987, 21, 229-231

6 Boulay, F.M., David, P.P. and Bourassa, M.G. Strategies for improving the work status of patients after coronary artery by-pass surgery Circulation 1982, 66(suppl III), 43-49

7 Gleichmann, U. Will there be less need for cardiac rehabilitation programmes when acute treatment is intensified and shortened? Eur Ht J 1987, 8(supp F), 29-33

8 Gloag, D. Rehabilitation of patients with cardiac conditions $B M J$ 1985, 290, 617-620

9 Mayou, R.A., MacMahon, D., Sleight, P., and Florencio, A.J. Early rehabilitation after myocardial infarction Lancet 1981, ii, 1399-1401

10 Vermeulen, A., Lie, K.I. and Durrer, M.D. Effects of cardiac rehabilitation after myocardial infarction: changes in coronary risk factors andlong term prognosis Am Heart J 1983, 105, 798-801

11 Waites, T.F., Watt, E.W. and Fletcher, G.F. Comparative functional and physiological status of active and dropout coronary bypass patients of a rehabilitation programme Am J Card 1983, 51, 1087-1090

12 Froelicher, V., Jensen, D., Genter, F. et al. A randomized trial of exercise trianing in patients with coronary heart disease JAMA 1984, 252, 1291-1297

13 Hoffmeister, J.M., Gruntizig, A.R. and Wenger, N.K. Long-term management of patients following successful percutaneous transluminal coronary angioplasty and coronary artery bypass grafting Cardiology 1986, 73, 323-332

14 CASS principal investigators. Coronary Artery Surgery Study (CASS): a randomized trail of coronary artery bypass surgery. Quality of life in patients randomly assigned to treatment groups Circulation 1983, 68,1 951-960

15 Bethell, H. How to do it: set up a coronary rehabilitation programme $B M J$ 1988, 297, 120-121 